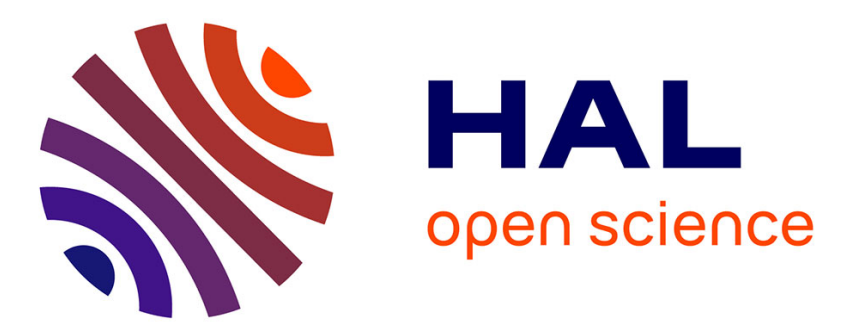

\title{
A substructuring approach for modeling the acoustic scattering from stiffened submerged shells coupled to non-axisymmetric internal structures
}

\author{
Valentin Meyer, Laurent Maxit, Christian Audoly
}

\section{- To cite this version:}

Valentin Meyer, Laurent Maxit, Christian Audoly. A substructuring approach for modeling the acoustic scattering from stiffened submerged shells coupled to non-axisymmetric internal structures. Journal of the Acoustical Society of America, 2016, 140 (3), pp.1609-1617. 10.1121/1.4962235 . hal-01468565

\section{HAL Id: hal-01468565 https://hal.science/hal-01468565}

Submitted on 16 Dec 2019

HAL is a multi-disciplinary open access archive for the deposit and dissemination of scientific research documents, whether they are published or not. The documents may come from teaching and research institutions in France or abroad, or from public or private research centers.
L'archive ouverte pluridisciplinaire HAL, est destinée au dépôt et à la diffusion de documents scientifiques de niveau recherche, publiés ou non, émanant des établissements d'enseignement et de recherche français ou étrangers, des laboratoires publics ou privés. 


\section{A substructuring approach for modeling the acoustic scattering from stiffened submerged shells coupled to non-axisymmetric internal structures}

Running title: Scattering from non-axisymmetric cylindrical submerged shells

Valentin Meyer ${ }^{a)}$, Laurent Maxit

INSA Lyon, Laboratoire Vibrations Acoustique, 25 bis, av. Jean Capelle, 69621 Villeurbanne, France

10

Christian Audoly

DCNS Research, 199 avenue Pierre-Gilles de Gennes, 83190 Ollioules, France

a) Author to whom correspondance should be addressed. Electronic mail: valentin.meyer@insa-lyon.fr 


\section{Abstract}

The scattered pressure from a stiffened axisymmetric submerged shell impinged by acoustic plane waves has been investigated experimentally, analytically and through numerical models. In the case where the shell is periodically stiffened, they show that helical, Bragg, and Bloch-Floquet waves can propagate. The influence of non-axisymmetric internal frames on the propagation of these waves is nevertheless not well known, as it can considerably increase the computational costs. To overcome this issue, the Condensed Transfer Function method, which has been developed to couple subsystems along linear junctions in the case of a mechanical excitation, is extended to acoustical excitations. It consists in approximating transfer functions on the junctions and to deduce the behavior of the coupled system thanks to the superposition principle and the continuity equations. In particular, it can be used to couple a dedicated model of an axisymmetric stiffened submerged shell with nonaxisymmetric internal structures modelled by the Finite Elements Method. Incident plane waves are introduced in the formalism and far-field re-radiated pressure is estimated. An application consisting in a stiffened shell with curved plates connecting the ribs is considered. Supplementary Bloch-Floquet trajectories are observed in the frequency-angle spectrum and are explained using a simplified interference model. 


\section{INTRODUCTION}

The scattering from a shell immersed in a heavy fluid and impinged by an acoustic plane wave has been intensively studied in the past. In addition to the specular reflection of the incident wave, the elastic shell vibrates and reradiates noise. The farfield scattered pressure shows typical patterns in the frequency-angle spectrum due to structural wave propagation and geometric diffraction. Compressional and shear waves that propagate helicoidally on the surface of the cylinder are highlighted using the elastic theory on infinite shells ${ }^{1 / 2}$ or on simply supported finite shells. $\frac{3}{3}$ For naval applications, cylinders are often reinforced by ring stiffeners or ribs to cope with the external static pressure. The influence of evenly spaced ribs was first investigated experimentally ${ }^{4}[6$ Two scattering phenomena are observed: the Bragg scattering, which is due to the interferences between the geometrical reflections of the incident plane wave on the ribs and the Bloch-Floquet scattering which is due to the subsonic flexural waves that propagate along the shell and interact with the internal frames. Tran-Van-Nhieu ${ }^{7}$ derives the problem analytically on a simply supported slender cylindrical shell, taking into account only the normal component of the reacting forces applied by the stiffeners to the shell. Guo distinguishes geometric and dynamic effect of an internal plate loading on an infinitely long cylinder, showing that resonances of the internal structure may affect greatly the scattered field. A similar 2D problem is analytically derived by Klauson and Metsaveer ${ }^{9}$ and they point out that lengthwise ribs and walls act on the circumferential waves. The inclusion of bulkheads is investigated in several studies ${ }^{10}$ which show that the coupling has an influence on the scattered pressure field. A quadrant symmetric arrangement of rods connected to the ring stiffeners through rubber blocks is considered by Park ${ }^{13}$ and he shows that the internal structures can store energy and lengthen the decay rate of an impulse reponse. Pan et al ${ }^{[14}$ describe the scattering from a finite cylindrical shell loaded by sets of stiffeners and bulkheads with two different spacings. They show that, in addition of the Bragg and Bloch-Floquet scattering due to the stiffeners, the effect of regularly spaced bulkheads can be seen. Experimental and numerical work are conducted to evaluate the effect of an increasing structural complexity of the submerged cylindrical shells: irregularly spaced ribs are considered, $\frac{15,16}{16}$ internal degrees of freedom are added through resonators, $\frac{17}{17}$ and the axisymmetry of the system is broken by internal structures 18 The theoretical formalism for these problems is given by Tran-Van-Nhieu 19 Several conclusions can be drawn from these studies. First the ribs spacing irregularity has mainly an influence on the near-field pressure, but can also modify the Bloch-Floquet and Bragg phenomena. Then, as mentioned by Soize, $\stackrel{20}{2}$ it shows that the internal fuzzy structures gives rise to an apparent damping effect. Finally, it can be said that breaking the axisymmetry increases the radiation efficiency of the structure.21

However, in industrial applications, internal substructures with various and complex geometries can be rigidly mounted on the stiffeners. Systems such as engine foundations can be found and one can wonder whether they play a role in the backscattered pressure. The experimental studies and semi-analytical methods presented so far are limited because of their low versatility, meaning that a dedicated set up or model should be created for each type 
of internal structure. That is why numerical methods need to be developed as an alternative to tackle this problem. In general, the element based methods, like for instance the Finite Element Method (FEM) or Boundary Element Method (BEM) are well suited for modeling systems with high geometry and property complexity. Due to the current computational limits, they are however limited to low frequencies when the system to be described is a submerged shell. To tackle this issue, Maxit proposes a sub-structuring approach called the Circumferential Admittance Approach (CAA) to describe the scattering of a submerged shell non periodically stiffened by internal frames ${ }^{22}$ It is based on the superposition principle for linear passive systems, where the admittances are defined on the circumferential orders, and allows modeling the vibroacoustic behavior on a large frequency range (typically up to $k a=40$, where $k$ is the acoustic wavenumber and $a$ the shell radius). This approach presents moreover several advantages: the cylindrical shell with heavy-fluid loading is described by a semi-analytical method that allows faster computation than usual discretization methods. Besides, stiffeners and bulkheads can be irregularly spaced and are modeled by FEM with shell elements. The stiffeners are thus not limited to 1D-beam models and radial, tangential, and axial efforts are taken into account along with the tangential moment. This method is nevertheless based on the assumption of an axisymmetric system, so that the circumferential orders can be studied separately one from the others.

Besides, a substructuring approach called the condensed transfer function (CTF) method has been recently developed to couple an axisymmetric stiffened submerged shell with nonaxisymmetric internal frames ${ }^{23}$ It consists in benefiting from the different methods proposed 95 above, by modeling the stiffened submerged shell by the CAA, whereas the internal frames are modeled by FEM, allowing flexibility on their geometry. In order to apply the CTF method, the junctions between the stiffened shell and the non-axisymmetric internal frames are supposed to be lines. Then, a set of orthonormal functions called condensation functions are used as a basis for approximating the displacements and the forces at the junctions. Condensed transfer functions are then defined and calculated for each uncoupled subsystems. The superposition principle for passive linear systems, the displacement continuity and the force equilibrium lead to the vibroacoustic behavior of the coupled system. However, the CTF method has so far only been applied to the case of mechanical point forces. In the present paper, the authors propose to extend the method to acoustical exciations, in order to study the scattering from non-axisymmetric submerged shells. The paper is organized as follows:

- The principle of the CTF method under an acoutical excitation in the case of a cylindrical shell is presented in section II.

- An application case is presented in section III. Numerical results are shown and physical phenomena are discussed.

- Conclusions are drawn in section IV. 


\section{COUPLING OF A STIFFENED SUBMERGED SHELL WITH NON-AXISYMMETRIC INTERNAL FRAMES}

\section{A Principle of the Condensed Transfer Function (CTF) method}

In this section, a substructuring method called the Condensed Transfer Function (CTF) method is introduced in the case of an acoustical excitation. As shown in Fig. 1, the system considered is made of an axisymmetric stiffened submerged cylindrical shell (subsystem 2), coupled to an internal non-axisymmetric frame (subsystem 1). An acoustic domain $\Omega$ is surrounding the cylindrical shell and $M$ is a point in the fluid domain. An acoustic plane wave impinges the shell in the vertical plane $\theta=0$, with a varying incidence $\alpha$ with regard to the normal to the shell. No fluid is considered inside the cylindrical shell.

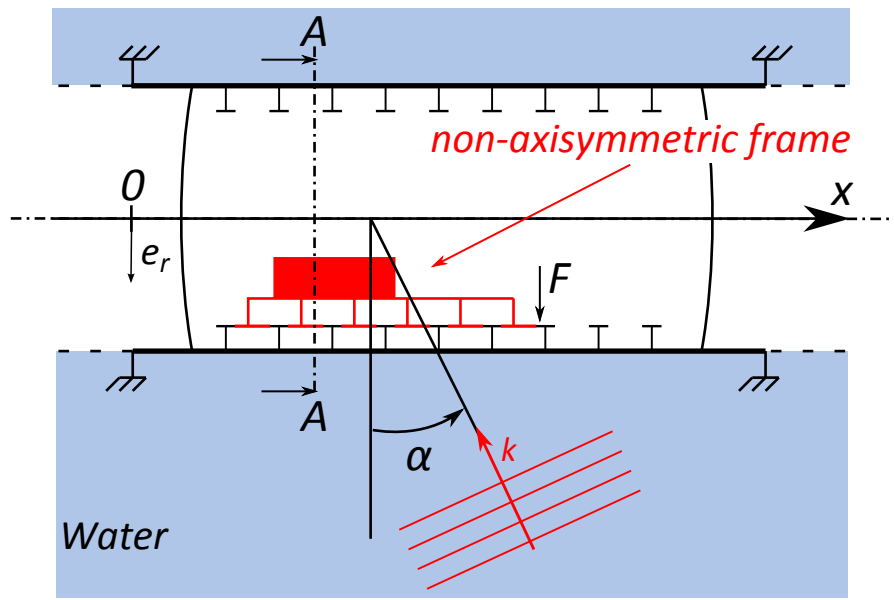

(a)

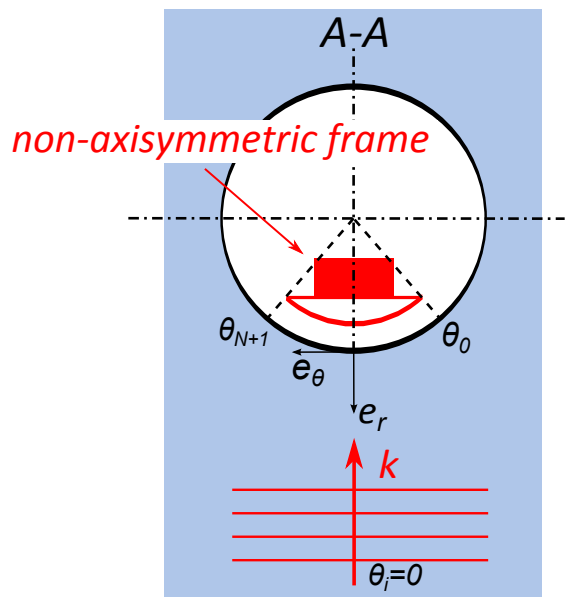

(b)

Figure 1: Sections of a stiffened cylindrical shell model including a non-axisymmetric internal frame and impinged by an oblique incident wave. (a) View in the plane $\theta=0$. (b) View in the plane $x=x_{A}$. (Color online)

The subsystems are modeled as thin mechanical structure such as plates or shells, so that the junctions between the two subsystems are lines. Considering here the cylindrical coordinates $(x, r, \theta)$, each junction between the subsystems is located at constant $x$ and $r$, and thus a point on the junction can be completely characterized by its coordinate $\theta$. $\Gamma$ represents the coupling line and the curvilinear abscissa $s$ is used to locate a point on $\Gamma$. The harmonic acoustic excitation is characterized by the blocked pressure, i.e. the pressure induced in the fluid when the system is considered as rigid ${ }^{24}$ The total pressure in the fluid medium $\Omega$ can then be decomposed as the sum of the blocked pressure $p_{b l}$ and the reradiated pressure $p_{r e}$ due to the system vibrations:

$$
p=p_{b l}+p_{r e}
$$


The aim is to calculate the reradiated pressure at the point $M$ when the two subssystems are coupled. To achieve this goal, the CTF (Condensed Transfer Function) approach previously developed in the case of mechanical excitations $\sqrt{23}[26$ is extended to calculate the reradiated pressure from informations calculated on the uncoupled sybsystems separatly. This method is based on the classical admittance (or mobility) method developed for point coupling. ${ }^{25}$

A set of $N$ orthonormal functions called the condensation functions is considered: $\left\{\varphi_{i}\right\}_{1 \leq i \leq N}$. They are defined on the junction $\Gamma$ and are functions of the curvilinear abscissa $s$. Examples of sets of condensation functions and the validation on the case of the vibrational behavior of a thin plate excited by a harmonic point load have been presented in an earlier study ${ }^{[26}$ An example of condensation functions which have shown good results and are easy to implement are the gate functions, defined according to their length $L_{s}$ as follows:

$$
\varphi_{i}(s)= \begin{cases}\frac{1}{\sqrt{L_{s}}} & \text { if }(i-1) L_{s} \leq s<i L_{s} \\ 0 & \text { elsewhere }\end{cases}
$$

This set consists in dividing the junction $\Gamma$ in $N$ segments of size $L_{s}$. The number of condensation functions $N$ plays a key role in the convergence of the method. Results show that $L_{s}$ should be smaller than half the smallest structural wavelength at the considered frequency to ensure good convergence. ${ }^{23}$ In the present case of a circular shell, the displacement at a point $s$ is written as a 4 components vector $U^{\alpha}(s)=\left(T_{x}, T_{r}, T_{\theta}, R_{\theta}\right)$, accounting for 3 translations and one rotation around the tangential coordinate. The rotations around the axial and radial coordinates can be deduced from the translations by the system continuity and are thus unnecessary in this formulation. Similarly, $F^{\alpha}(s)$ is a 4 components vector accounting for the forces in the 3 directions and the moment around the tangential coordinate.

The principle of the CTF method consists in approximating the displacements vector $U^{\alpha}(s)$ and the forces vector $F^{\alpha}(s)$ at the junction as a linear combination of these condensation functions for each subsystem $\alpha \in\{1,2\}$ :

$$
\left\{\begin{array} { l } 
{ U ^ { 1 } ( s ) \simeq \sum _ { i = 1 } ^ { N } u _ { i } ^ { 1 } \varphi _ { i } ( s ) } \\
{ U ^ { 2 } ( s ) \simeq \sum _ { i = 1 } ^ { N } u _ { i } ^ { 2 } \varphi _ { i } ( s ) }
\end{array} \text { and } \left\{\begin{array}{l}
F^{1}(s) \simeq \sum_{i=1}^{N} f_{i}^{1} \varphi_{i}(s) \\
F^{2}(s) \simeq \sum_{i=1}^{N} f_{i}^{2} \varphi_{i}(s)
\end{array}\right.\right.
$$

where $u_{i}^{\alpha}$ (resp. $f_{i}^{\alpha}$ ) is the displacement amplitude vector (resp. the force amplitude vector) of subsystem $\alpha$ associated to the condensation function $\varphi_{i}$.

For the uncoupled subsystems, the following quantities need to be calculated:

1. The condensed transfer function between $\varphi_{i}$ and $\varphi_{j}$, defined by applying a force $F^{\alpha}=$ $\varphi_{j}$ on $\Gamma$ :

$$
Y_{i j}^{\alpha}=\frac{\left\langle\bar{U}_{j}^{\alpha}, \varphi_{i}\right\rangle}{\left\langle F^{\alpha}, \varphi_{j}\right\rangle}=\left\langle\bar{U}_{j}^{\alpha}, \varphi_{i}\right\rangle
$$

where $\bar{U}_{j}^{\alpha}$ is the displacement of the junction $\Gamma$ when the subsystem $\alpha$ is excited by 160 $F^{\alpha}=\varphi_{j}$, and $\langle\bullet, \bullet\rangle$ is a scalar product defined in the case of gate functions by

$$
\begin{aligned}
\mathcal{C}_{I}^{0} \times \mathcal{C}_{I}^{0} & \rightarrow \mathbb{C} \\
\langle f, g\rangle & \mapsto \int_{\Gamma} f(s) g^{*}(s) \mathrm{d} s
\end{aligned}
$$


where $\mathcal{C}_{I}^{0}$ is the set of piecewise continuous functions on intervals $[a, b[\subset \Gamma$, and * means the complex conjugate. It can be easily verified that the gate functions form an orthonormal set for this scalar product. For a couple $(i, j), Y_{i j}^{\alpha}$ is a $4 \times 4$ matrix that describes the couplings between the condensed physical quantities for all degrees of freedom.

2. The condensed transfer function between $\varphi_{i}$ and an observation point $M$ on subsystem 2 is defined by

$$
Y_{M i}^{2}=P_{i}^{2}(M), \forall i \in\{1,2, \ldots, N\}
$$

where $P_{i}^{2}(M)$ is the pressure at the point $M$ when the subsystem 2 is uncoupled and excited by $F^{2}=\varphi_{i}$ on $\Gamma$. As the force can be applied in one of the 4 directions, $Y_{M i}^{2}$ is a 4 components vector.

3. The free condensed displacements vector of each uncoupled subsystem $\alpha$ are defined by:

$$
\tilde{u}_{i}^{\alpha}=\left\langle\tilde{U}^{\alpha}, \varphi_{i}\right\rangle, \forall i \in\{1,2, \ldots, N\}
$$

where $\tilde{U}^{\alpha}$ is the displacement at the junction of the uncoupled subsystem $\alpha$ when only external loading is applied. As in the example of Fig. 1 the subsystem 1 has no external load, then: $\tilde{u}_{i}^{1}=0, \forall i \in\{1,2, \ldots, N\}$.

In response to the line coupling forces and to the external load, the superposition principle for passive linear systems enables to write the displacements coefficients $u_{i}^{\alpha}$ as follows:

$$
\left\{\begin{array}{c}
u_{i}^{1}=\tilde{u}_{i}^{1}+\sum_{j=1}^{N} Y_{i j}^{1} f_{j}^{1} \\
u_{i}^{2}=\sum_{j=1}^{N} Y_{i j}^{2} f_{j}^{2}
\end{array} \quad, \forall s \in \Gamma, \forall i \in\{1,2, \ldots, N\}\right.
$$

Besides, the displacement continuity and the force equilibrium equation at the junction lead to

$$
\left\{\begin{array}{c}
U^{1}(s)=U^{2}(s) \\
F^{1}(s)+F^{2}(s)=0
\end{array}, \forall s \in \Gamma\right.
$$

The set of condensation functions being orthonormal, the projection of Eq. (9) on a function $\varphi_{i}$ yields

$$
\left\{\begin{array}{c}
u_{i}^{1}=u_{i}^{2} \\
f_{i}^{1}=-f_{i}^{2}
\end{array} \quad, \forall i \in\{1,2, \ldots, N\}\right.
$$

The coupling forces $\mathbf{F}^{\mathbf{c}}=\mathbf{F}^{\mathbf{1}}=-\mathbf{F}^{\mathbf{2}}$ between the subsystems is deduced by injecting Eq. (8) into Eq. (10):

$$
\left(\mathbf{Y}^{1}+\mathbf{Y}^{2}\right) \mathbf{F}^{c}=\tilde{\mathbf{U}}^{2}
$$

where $\mathbf{Y}^{\mathbf{1}}$ (resp. $\mathbf{Y}^{\mathbf{2}}$ ) is the condensed transfer function matrix of subsystem 1 (resp. subsystem 2) and is built by making $i$ and $j$ vary in Eq. (4), resulting in a $4 N$ square matrix. Similarly, $\tilde{\mathbf{U}}^{2}$ is the free condensed displacements vector where each element is given by Eq. (7). 
Once the coupling forces have been calculated by inverting Eq. (11), the pressure at the point $M$ of the coupled system can be deduced:

$$
p_{r e}(M)=\tilde{p}_{r e}^{2}(M)-\sum_{i=1}^{N} Y_{M i}^{2} F_{i}^{c}
$$

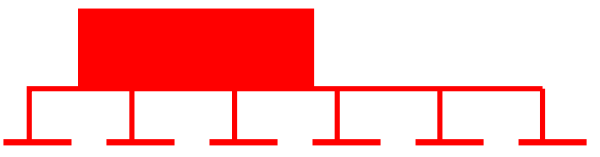

(a)

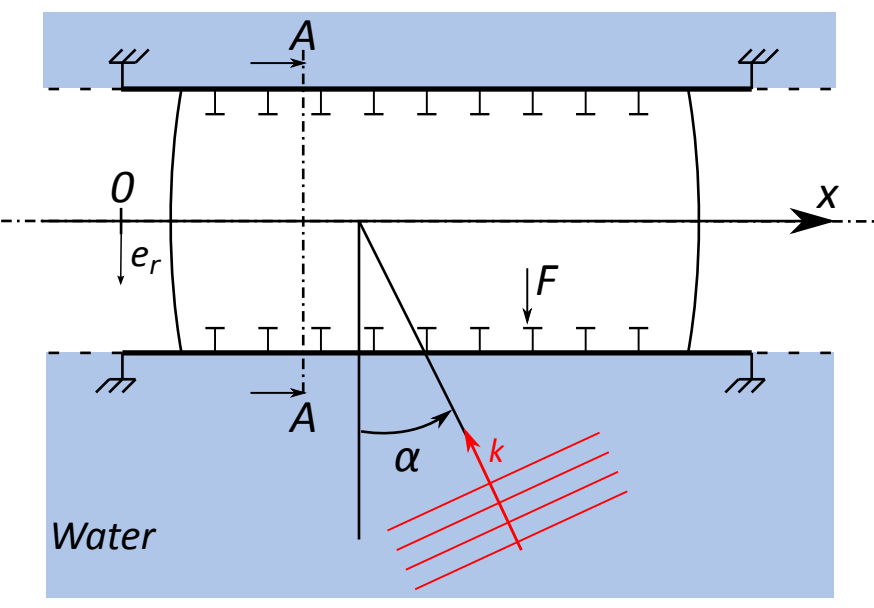

(b)

Figure 2: Sections in the plane $\theta=0$ of (a) a non-axisymmetric internal frame and (b) an axisymmetric stiffened cylindrical shell model. (Color online)

Subsystem 1 (the non-axisymmetric frame shown in Fig. 1) is modeled by the Finite Element Method (FEM) and its condensed transfer functions are calculated by applying a force $\varphi_{j}$ on the junction and projecting the resulting displacement of the junction on $\varphi_{i}$, for all combinations of $(i, j) \in\{1,2, \ldots, N\}^{2}$ (see Eq. (4)). Regarding computational costs, as subsystem 1 is uncoupled and has no fluid loading, the Finite Element analysis can be carried out on a wide frequency band, provided that the mesh is fine enough.

Subsystem 2 is described by the Circumferential Admittance Approach (CAA), which is a dedicated method to predict the vibroacoustic behavior of a submerged shell with internal 
axisymmetric frames. The approach consists in partitioning the axisymmetric system and considering the submerged shell separately from the internal frames (ring stiffeners, bulkheads, end caps). It has been first introduced in the case of a point harmonic force $e^{28}$ and extended to model the sound scattering. ${ }^{22}$ All the physical variables (i.e. efforts, shell displacements, and pressure) can be written as Fourier series depending on circumferential orders $k_{\theta}$ :

$$
f(\theta)=\sum_{k \in \mathbb{Z}} \tilde{f}\left(k_{\theta}\right) \mathrm{e}^{\mathrm{j} k_{\theta} \theta}
$$

where $k_{\theta} \in \mathbb{Z}$, because the system is $2 \pi$-periodic along the circumference, and with

$$
\tilde{f}\left(k_{\theta}\right)=\frac{1}{2 \pi} \int_{-\pi}^{\pi} f(\theta) \mathrm{e}^{-\mathrm{j} k_{\theta} \theta} \mathrm{d} \theta
$$

The shell and the frames are supposed to be axisymmetric and the fluid domain is infinite, so that the circumferential orders are independent one from the others. The circumferential admittances of the fluid loaded shell are estimated using the Flügge equations of motions and by solving the problem in the wavenumber space. Different types of ring stiffeners can be modeled using axisymmetric FEM. This strategy enables to save computational costs because the fluid loaded is solved semi-analytically and the stiffeners have no fluid loading and are of reasonable size regarding the current computational capacities. The coupling forces $\tilde{\mathbf{F}}^{\text {frame }}$ between the shell and the stiffeners are then calculated thanks to the circumferential admittances of the subsystems $\tilde{\mathbf{Y}}^{\text {shell }}$ and $\tilde{\mathbf{Y}}^{\text {frame }}$ and to the free displacements of the shell $\tilde{\overline{\mathbf{W}}}^{\text {shell }}$, by inverting the following equation:

$$
\left[\tilde{\mathbf{Y}}^{\text {shell }}+\tilde{\mathbf{Y}}^{\text {frame }}\right] \tilde{\mathbf{F}}^{\text {frame }}=-\tilde{\overline{\mathbf{W}}}^{\text {shell }}
$$

The free displacements are calculated from the Flügge equations using on the right hand term the blocked pressure due to the oblique plane wave:22]

$$
p_{b l}(x, r, \theta)=e^{\mathrm{j} \overline{\mathrm{k}}_{x} x} \sum_{n=-\infty}^{+\infty} \bar{p}_{b l, n}(r) e^{\mathrm{j} n \theta}
$$

where $\bar{k}_{x}=k_{0} \sin \alpha$, and:

$$
\bar{p}_{b l, n}(r)=p_{0 \mathrm{j}}{ }^{n}\left[J_{n}\left(\bar{k}_{r} r\right)-\frac{1}{\cos \alpha} \frac{J_{n}^{\prime}\left(\bar{k}_{r} R\right)}{H_{n}^{(2)^{\prime}}\left(\bar{k}_{r} R\right)} H_{n}^{(2)}\left(\bar{k}_{r} r\right)\right]
$$

with $p_{0}$ the amplitude of the incident pressure, $R$ the radius of the shell, $\bar{k}_{r}=k_{0} \cos \alpha, J_{n}$ the Bessel function of first kind of order $n$ and $H_{n}^{(2)}$ the Hankel function of the second kind of order $n$. The forces are finally injected in the shell model to deduce the shell displacements. The stationary phase theorem is used to calculate the far-field reradiated pressure.

The axisymmetry of subsystem 2 is used in order to calculate its condensed transfer functions more efficiently. As only the tangential coordinate $\theta$ is supposed to vary along the 
junctions, it is thus sufficient to calculate the reponse $U_{0}^{2}$ to a point force on the junction (for instance for $\theta=0$ ) and deduce the reponse $\bar{U}_{j}^{2}$ to a condensed force $\varphi_{j}$ by convolution:

$$
\bar{U}_{j}^{2}(\theta)=\int_{\theta_{0}}^{\theta_{N+1}} U_{0}^{2}(\theta-\alpha) \varphi_{j}(\alpha) \mathrm{d} \alpha
$$

The condensed transfer functions are then calculated by projecting this reponse on $\varphi_{i}$ (see Eq. (41)):

$$
\begin{aligned}
Y_{i j}^{2} & =\frac{\left\langle\bar{U}_{j}^{2}(\theta), \varphi_{i}(\theta)\right\rangle}{\left\langle\varphi_{j}(\theta), \varphi_{j}(\theta)\right\rangle} \\
& =\left\langle\int_{\theta_{0}}^{\theta_{N+1}} U_{0}^{2}(\theta-\alpha) \varphi_{j}(\alpha) \mathrm{d} \alpha, \varphi_{i}(\theta)\right\rangle
\end{aligned}
$$

Besides, the displacements of the axisymmetric stiffened shell impinged by an oblique incident wave are also calculated through the CAA method, as explained in.22 The free condensed displacements (right-hand term in Eq. (11)) are deduced by projecting this reponse on $\varphi_{i}$ (see Eq. (7)).

Once the condensed transfer functions have been calculated for each subsystem, the coupling forces at the junction between the axisymmetric stiffened shell and the non-axisymmetric internal frame are calculated by inverting Eq. (11). In the final step, the pressure scattered from the whole system is deduced thanks to Eq. (12). In this equation, $\tilde{p}_{r e}^{2}(M)$ and $Y_{M i}^{2}$ have been calculated with the CAA in the same step than the CTF calculation of subsystem 2 . Hence, if these terms are stored in a database, no extra calculations are needed.

\section{TEST CASE APPLICATION}

\section{A Description of the system}

In this section, non-axisymmetric internal frames are added to the stiffened shell experimentally investigated by Liétard et al., ${ }^{\sqrt[6]{6}}$ and numerically modeled by Maxit. ${ }^{22}$ The system is modeled by a $750 \mathrm{~mm}$ long simply-supported cylindrical shell with a radius of $a=50 \mathrm{~mm}$ and a thickness of $h=1 \mathrm{~mm}$. It is stiffened by 49 evenly spaced internal rings having $5 \mathrm{~mm}$ $\times 1 \mathrm{~mm}$ rectangular cross-sections. The stiffeners spacing is $d=15 \mathrm{~mm}$. The system is made of steel $\left(E=2.4 \times 10^{11} \mathrm{~Pa}, \rho=7900 \mathrm{~kg} \cdot \mathrm{m}^{-3}, \nu=0.3\right)$ and immersed in water $\left(\rho_{0}=1000\right.$ $\left.\mathrm{kg} . \mathrm{m}^{-3}, c_{0}=1470 \mathrm{~m} . \mathrm{s}^{-1}\right)$. A structural damping coefficient of $\eta=0.2 \%$ is accounted for as a complex factor in the stiffness matrix.

48 curved plates $\left(15 \times 73 \mathrm{~mm}^{2}, 1 \mathrm{~mm}\right.$ thick, curvature radius of $r_{i n t}=45 \mathrm{~mm}$, represented by the mesh in Fig (3) are added at the tip of the stiffeners, on the whole length of the shell, as shown in Fig. 3a. This results in 47 junctions between one rib and two plates and 2 junctions between one rib and one plate at the extremities. These plates are nonaxisymmetric and extend from $\theta=-46^{\circ}$ to $\theta=46^{\circ}$, covering thus approximately one fourth of the circumference. A plane wave impinges the system in a manner that it remains symmetric (i.e. in the plane $\theta=0$, coming from under the shell in Fig. 1 and 3 a). 


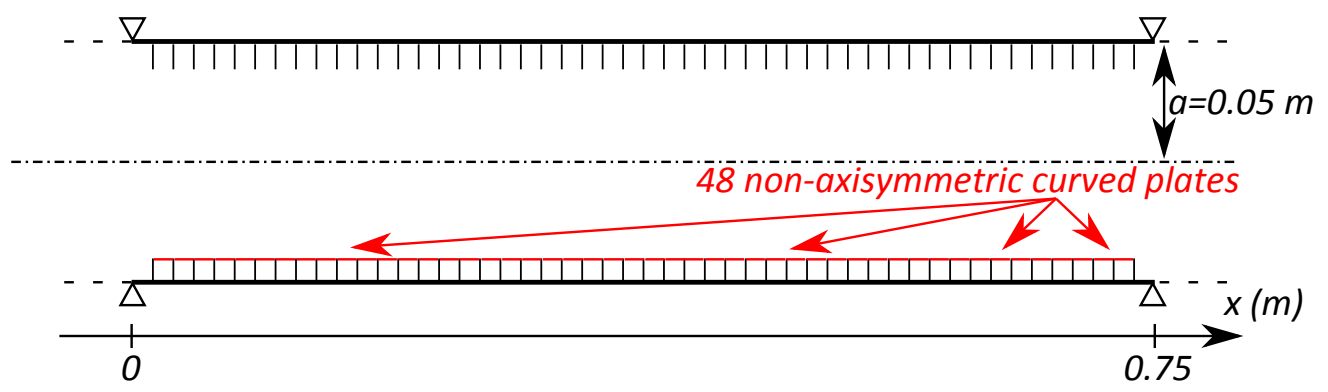

(a)

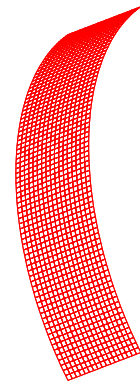

(b)

Figure 3: Submerged hull. (a) Cylindrical shell: $50 \mathrm{~mm}$ radius, $0.75 \mathrm{~m}$ length, $1 \mathrm{~mm}$ thick. Sitffeners: spacing $15 \mathrm{~mm}$, rectangular cross-section $(\mathrm{mm}): 5 \times 1$ (a) Section in the plane $\theta=0$. (b) FEM model of one curved plate.

According to the convergence criterion defined in,, 26 the length of the gates $L_{s}$ is chosen shorter than half the smallest flexural wavelength in the frequency range. Considering frequencies up to $200 \mathrm{kHz}(k a \simeq 42)$ this criterion allows a maximum gate length of $3.6 \mathrm{~mm}$ for the present case. As the coupling junction between a rib and the curved plates is $73 \mathrm{~mm}$ long, $N=20$ condensation functions are then considered.

The FE model used for calculating the condensed transfer functions of the plates is made of quadrilateral isotropic shell elements. The mesh is fine enough to respect the criterion of 6 elements per bending wavelength at the highest frequency. As the 48 plates are identical, it is sufficient to calculate the condensed transfer functions on one plate and use them for all the junctions. Details on the parameters for the CAA calculation are found in. ${ }^{22}$ It is worth noting that the Love's first approximations for shells are still valid at high frequencies and the shell theories can thus still be used. $\stackrel{29}{ }$

In section III, the process to couple an axisymmetric shell described by CAA with nonaxisymmetric internal structures described by FEM has been explained. The sketch in Fig. 4 summarizes the different steps and gives calculation times for each step for the test case used in this section. Given calculation times correspond to the frequency of $200 \mathrm{kHz}$, and for all incidence angles $\alpha$ varying between 0 and $179^{\circ}$ with a step of $1^{\circ}$. The method is coded in Matlab. FEM is used to calculate the internal structures admittances: NASTRAN for the axisymmetric ribs and the SDT Structural Dynamics Toolbox for the non-axisymmetric internal frames. Calculations are performed on a 64 Gb RAM computer. Fig. 4 shows that the calculation for the axisymmetric case takes approximately 15 minutes (the response to mechanical excitations is only necessary to calculate the condensed transfer functions for the coupling). As quantities are stored in a database, taking into account the 48 nonaxisymmetric plates adds less than 32 minutes of calculation, that being three times more than the axisymmetric case. This time can however be shortened by parallelizing some steps that are independent, as for instance the response of the axisymmetric subsystem to acoustical and mechanical excitations.

No comparison can be proposed by classical numerical methods such as FEM-BEM, 


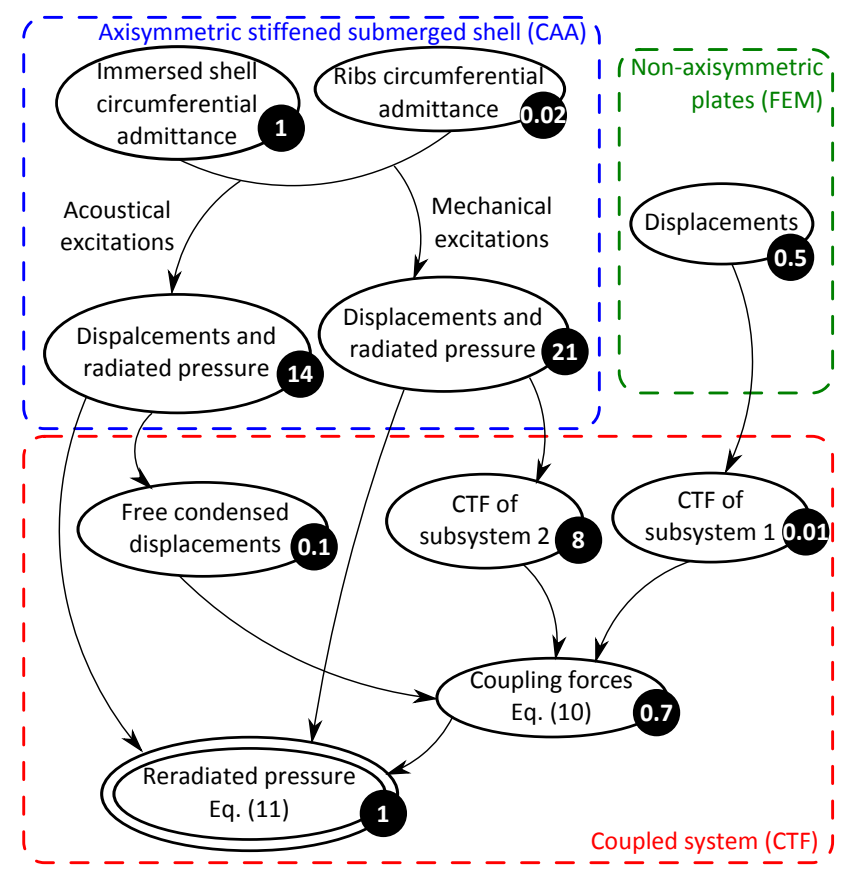

Figure 4: Sketch of the method to account for non-axisymmetric internal frames in a non periodically stiffened submerged shell, with corresponding calculation times (in minutes).

regarding the size of the whole system and the frequencies of interest. Nevertheless, the CTF method has been previously validated, ${ }^{23 \mid 26}$ and the originality of this work consists in replacing the free displacement in the right hand side of Eq. (11), which does not affect the validity of the method. On the other hand, the free displacement calculation has also been validated 22

\section{B Influence of the non-axisymmetric internals on the backscatter- ing}

The reradiated component of the backscattered pressure from the stiffened shell, without the non-axisymmetric internal frames, is plotted on Fig. 5a as a function of the dimensionless frequency $k a$ and the incidence angle $\alpha$. Higher values of backscattered pressure are due to helical waves in the case of low incidence angles $\left(\alpha<30^{\circ}\right)$ and for $k a \in[0,30]$. High values of pressure can also be seen because of Bloch-Floquet and Bragg scattering, for which the incidence angle decreases with the frequency. These two phenomenon are due to the regularly spaced ribs. Bragg scattering is the result of interferences of the geometrical reflections of the incident plane wave on the rings. Bloch-Floquet scattering results from the quasi-flexural waves which propagates along the shell and interacts with the ribs. ${ }^{6}$

The influence of the non-axisymmetric internals on the backscattered pressure spectrum is evaluated through the method presented in section [I and the results are plotted in Fig. 5b. Differences can be seen on the backscattered spectrum and the following comments can be 


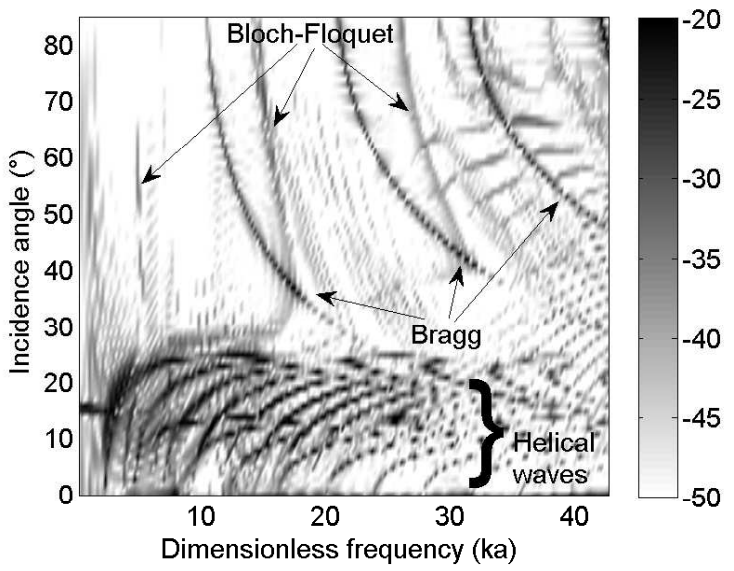

(a)

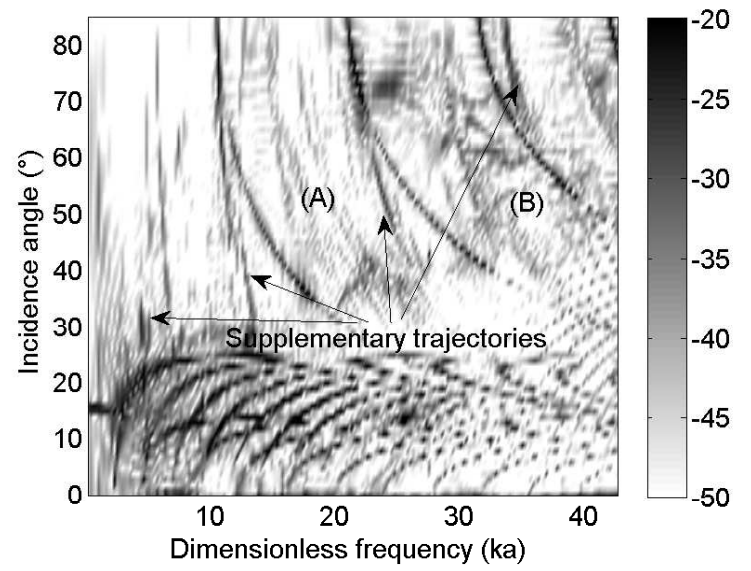

(b)

Figure 5: (a) Spectrum of monostatic backscattered pressure of the axisymmetric shell. ${ }^{22}$ (b) Spectrum of the shell including the non-axisymmetric internal frames.

made:

- The propagation of helical waves on the shell is not significantly affected by the presence of the internal frames.

- Similarly, as the regularly spaced ribs have not been modified, the Bragg scattering due to geometrical reflections can still be clearly seen.

- Supplementary trajectories, that can be apparented to Bloch-Floquet scattering, can be observed. A focus on this phenomenon is proposed in the next section. In the following, the adjective "standard" refers to the Bloch-Floquet waves seen in the axisymmetric case, whereas "supplementary" refers to the trajectories marked in Fig. 5b and that are due to the internal structures.

- In the areas marked (A) and (B), standard Bloch-Floquet waves that are visible in the axisymmetric case cannot longer be clearly seen. This can be explained by the apparition of supplementary vibration energy propagation paths that reduces the ones at the origin of the standard Bloch-Floquet waves.

In the CAA, the circumferential orders are calculated separately and the quantities $\tilde{p}_{r e}^{2}(M)$ and $Y_{M i}^{2}$ can be expressed as Fourier series. Hence, the left hand term of Eq. (12) can also be written as a Fourier serie. In this sense, it is possible to isolate the contribution of a given circumferential order. For $k_{\theta}=4$ for isntance, only two trajectories related to helical waves can be seen for the axisymmetric case: one due to shear waves and the other one to compressional waves, as shown in the upper part of Fig 6. This spectrum can be compared to the non-axisymmetric case in the lower part of Fig 6. The dominant trajectories correponding to the shear and compressional helical waves are the same than in the axisymmetric case. 
Nevertheless, additional trajectories can be seen between the two dominant trajectories. This observation highlights that there is a coupling between the circumferential orders due to the non-axisymmetry. This result is consistent with the litterature, $\frac{18[21] 23}{23}$ and can also explain why the patterns are more complex in the areas marked (A) and (B) in Fig. 5 b.

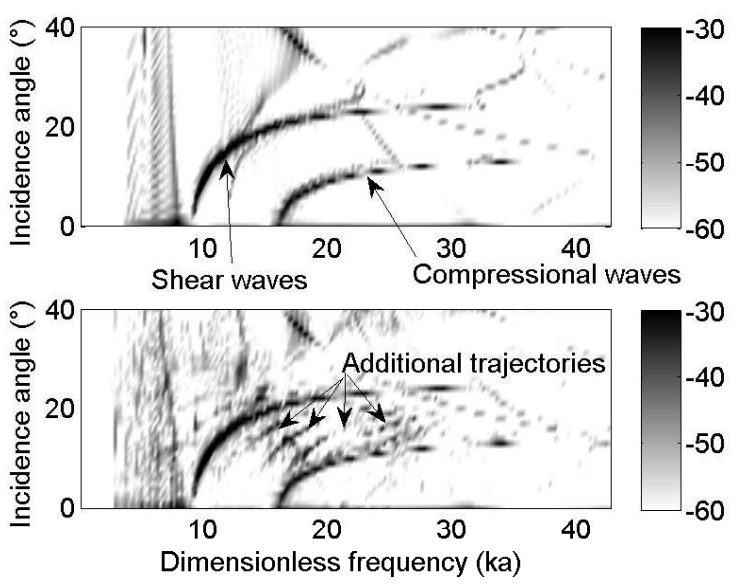

Figure 6: Spectrum of monostatic backscattered pressure for the circumferential order $k_{\theta}=4$. Upper part: axisymmetric shell. Lower part: non-axisymmetric shell.

\section{Discussion on the supplementary trajectories}

In order to take a close look on the supplementary trajectories marked in Fig. 55b, only the contribution of the circumferential order $k_{\theta}=1$ is plotted in Fig. 7 a. According to their shape and to the litterature,,$[6]$ these supplementary trajectories are apparented to Bloch-Floquet scattering and are marked BF (F) for the forward waves and BF (B) for the backward waves. At high incidence $\left(\alpha \sim 80^{\circ}\right)$, the Bloch-Floquet forward waves appear at the same frequency than for Bragg scattering. This observation happens to be a simple geometrical coincidence but is helpful to check the trajectories locations on the frequency-angle spectrum.

To analyze more in details the phenomenon, a simple scattering/interference model based on the formulation given in ${ }^{6}$ is developed. The sketch in Fig. 8 shows that in addition of the propagation wave along the shell, the flexural waves can propagate in the internal structures. Indeed, an incoming wave impinges the shell on a stiffener $n$, creates a structural vibration that can propoagate through the stiffeners and the curved plate to finally reradiate through the shell at another stiffener $m$. Taking into account that all the stiffeners are excited by the acoustic wave, the trajectories of this Bloch-Floquet scattering is thus given by the following double summation:

$$
P^{B F}=P_{0} \sum_{n=1}^{49} e^{\mathrm{j} 2 k(n-25) d \sin \alpha} \sum_{m=1}^{49} e^{\mathrm{j} \Psi_{m n}}
$$

with

$$
\Psi_{m n}=(m-n)\left(\operatorname{sgn}(m-n) k_{x f}\left(2 h_{w}+d\right)+k d \sin \alpha\right)
$$




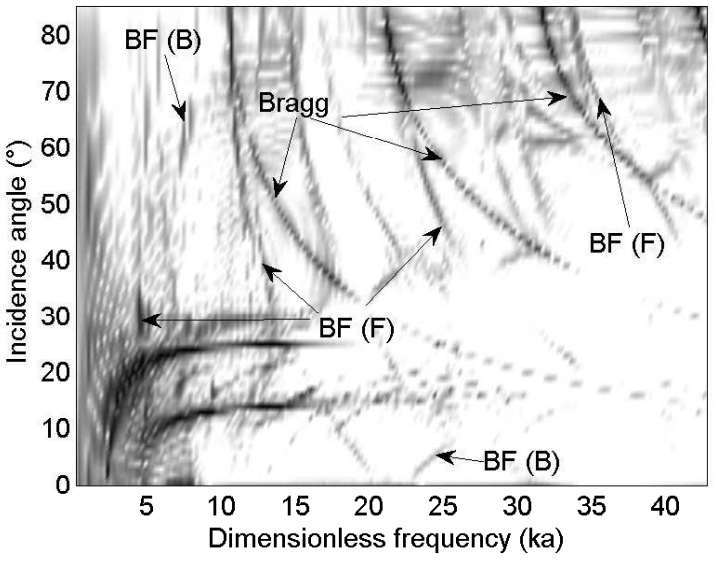

(a)

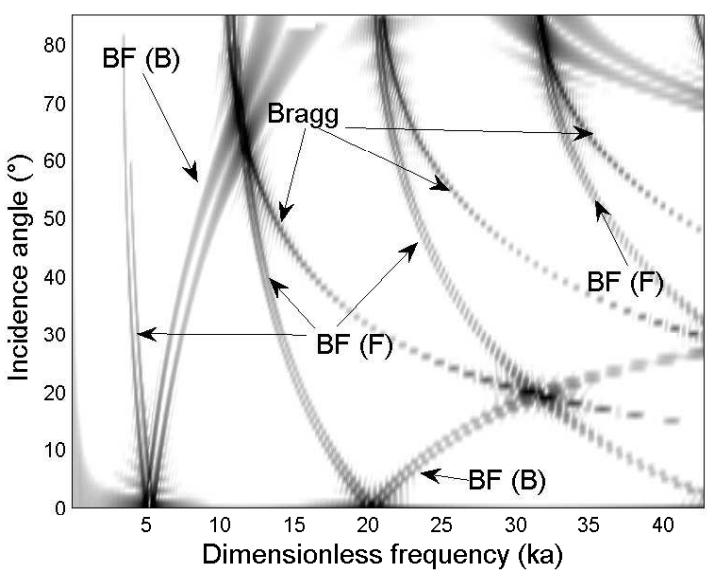

(b)

Figure 7: (a) Spectrum of monostatic backscattered pressure of the non-axisymmetric shell for the circumferential order $k_{\theta}=1$ only. (b) Trajectories of Bragg and Bloch-Floquet waves using the simplified interference model.

where $x \mapsto \operatorname{sgn}(x)$ is the sign function, $h_{w}$ is the height of a stiffener, and $k_{x f}$ is the wavenumber of the flexural waves in the internals, decomposed in the plane $\theta=0 . k_{x f}$ depends on the circumferential order $k_{\theta}$ and on the flexural wavenumber of an equivalent in vacuo plate $k_{f}$ :

$$
k_{x f}\left(k_{\theta}\right)=\sqrt{k_{f}^{2}-\left(\frac{k_{\theta}}{R}\right)^{2}}
$$

Plotting these equations for $k_{\theta}=\{1,4\}$ with a normalized amplitude gives the spectrum in Fig. 7b. The standard Bloch-Floquet waves due to the waves propagating on the shell are not represented for the sake of clarity, but Bragg scattering is kept as a point of reference on the frequency-angle spectrum. The fourth circumferential order has been chosen because it might play a particular role considering that the curved plates cover one fourth of the circumference. The flexural wavenumber $k_{f}$ at a frequency $f$ is calculated through the following formula:24

$$
k_{f}=\sqrt{\frac{2 \pi f}{h^{\prime}}}\left(\frac{12\left(1-\nu^{2}\right) \rho}{E}\right)^{1 / 4}
$$

where $h^{\prime}$ is a corrected thickness that accounts for the fact that the plates are stiffened by the ribs and is calculated by averaging the material added by the stiffeners along the shell, resulting in $h^{\prime} \simeq 1.3 \mathrm{~mm}$.

Comparing Fig. $7 \mathrm{~b}$ and Fig. $7 \mathrm{~b}$ show satsifying results. Indeed, the locii of the supplementary Bloch-Floquet trajectories evaluated with the model match the ones on the numerical simulations. Slight shifts can however be observed, especially at high frequencies. This is explained by the fact that the interference model is built to give trends and is highly sim- 


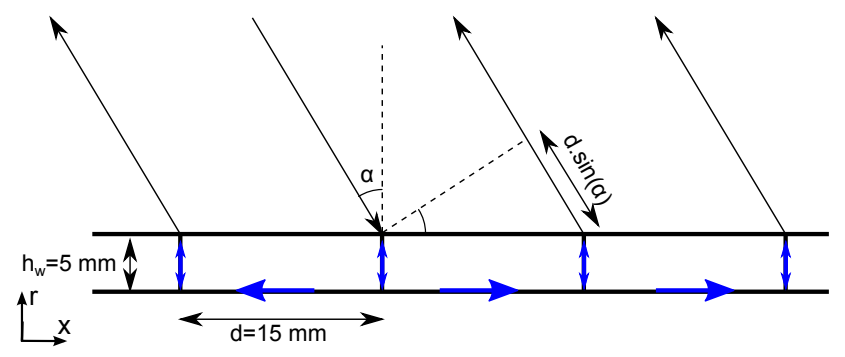

Figure 8: Sketch of the insonified shell and of the propagation of flexural waves in the internal structures. (Color online)

plified. More precisely, it is not able to accurately account for the all the couplings between the shell, the ribs, and the internal plates.

Supplementary simulations have been conducted on modified geometries to check the assumptions made in this section. The spectra are not presented for the sake of brievity but the following comments can be made and prove that the supplementary trajectories only depends on the internals geometrical parameters:

- If the shell thickness is doubled, the supplementary Bloch-Floquet trajectories are not modified. Bragg scattering is also unchanged but the locations of the standard BlochFloquet waves traveling on the shell are changed.

- If the plates length is reduced (covering only one eight of the circumference for instance), the supplementary Bloch-Floquet trajectories are the same but can be seen less clearly.

- If the internal plates thickness is changed, the supplementary trajectories are no longer visible. In this case, the plates thickness is no longer the same than the ribs thickness. Hence, there is an impedance mismatch between the stiffened shell and the internal structures, and the waves cannot propagate easily.

\section{CONCLUDING REMARKS}

The substructuring CTF method has been presented to predict numerically the scattering from a stiffened submerged shell with non-axisymmetric internal frames. As only condensed transfer functions at the junctions between the subsystems are needed, one of the main advantages of this method is that it is able to couple subsystems that are described by different approaches. In this paper, the stiffened shell has been described by the Circumferential Admittance Approach while the internal frames by the Finite Element Method. As the FEM offers great flexibility on the geometrical design, any non-axisymmetric internal frame coupled along a line junction can be taken into account by the CTF method. The method is however more efficient for junctions where the axial and radial coordinates remain constant in order to benefit from the axisymmetric properties of the stiffened shell. Two other advantages of the CTF approach is that it can reach high frequencies $(k a>40)$ and that the results can be directly investigated in the wavenumber space. 
400

Through a particular example of an immersed shell with internal plates joining adjacent stiffeners all along the shell. It has been shown that the non-axisymmetric frames have an influence on the backscattered spectrum. Indeed, the Bloch-Floquet scattering is harder to identify than in the axisymmetric case. Moreover, supplementary trajectories apparented to Bloch-Floquet scattering, for which waves propagate through the non-axisymmetric internal structures, have been pointed out and explained through a simple interference model. These trajectories highly depend on the geometry of the internal structures, but it shows that scattering due to flexural waves traveling inside the shell can be seen in the far-field. As the developed model presents high versatiliy concerning the design of the internal structures, further work consists in modeling cases of internal structures taken from industrial applications and investigate their effect on the scattered pressure field. Moreover, as the modeling of the internal structure is carried out independantly from the stiffened shell modeling for the calculation of the CTF, this approach is well adapted to optimize internal structures with regard to the backscattered pressure.

\section{Acknowledgment}

45 This work was funded by DCNS and performed within the framework of the LabEx CeLyA of Université de Lyon, operated by the French National Research Agency (ANR-10-LABX0060/ANR-11-IDEX-0007).

\section{References}

${ }^{1}$ L. Flax, V. K. Varadan, and V. V. Varadan, "Scattering of an obliquely incident acoustic wave by an infinite cylinder," J. Acoust. Soc. Am. 68, 1832-1835 (1980).

${ }^{2}$ F. Léon, F. Lecroq, D. Décultot, and G. Maze, "Scattering of an obliquely incident acoustic wave by an infinite hollow cylindrical shell," J. Acoust. Soc. Am. 91, 1388-1397 (1992).

${ }^{3}$ M. L. Rumerman, "Contribution of membrane wave reradiation to scattering from finite cylindrical steel shells in water," J. Acoust. Soc. Am. 93, 55-65 (1993).

${ }^{4}$ D. M. Photiadis, J. A. Bucaro, and B. H. Houston, "Scattering from flexural waves on a ribbed cylindrical shell," J. Acoust. Soc. Am. 96, 2785-2790 (1994).

${ }^{5}$ B. H. Houston, J. A. Bucaro, and D. M. Photiadis, "Broadband acoustic scattering from a ribbed shell," J. Acoust. Soc. Am. 98, 2851-2853 (1995).

${ }^{6}$ R. Liétard, D. Décultot, G. Maze, and M. Tran-Van-Nhieu, "Acoustic scattering from a finite cylindrical shell with evenly spaced stiffeners: Experimental investigation," J. Acoust. Soc. Am. 118, 2142-2146 (2005).

${ }^{7}$ M. Tran-Van-Nhieu, "Scattering from a ribbed finite cylindrical shell," J. Acoust. Soc. Am. 110, 2858-2866 (2001). 
${ }^{8}$ Y. P. Guo, "Sound scattering from cylindrical shells with internal elastic plates," J. Acoust. Soc. Am. 93, 1936-1946 (1993).

${ }^{9}$ A. Klauson and J. Metsaveer, "Sound scattering by a cylindrical shell reinforced by lengthwise ribs and walls," J. Acoust. Soc. Am. 91(4):1834-1843 (1992).

${ }^{10}$ Y. P. Guo, "Sound scattering by bulkheads in cylindrical shells," J. Acoust. Soc. Am. 95, 2550-2559 (1994).

${ }^{11}$ J. M. Cuschieri and D. Feit, "Acoustic scattering from a fluid-loaded cylindrical shell with discontinuities: Single plate bulkhead," J. Acoust. Soc. Am. 98, 320-338 (1995).

12 J. M. Cuschieri and D. Feit, "Acoustic scattering from a fluid-loaded cylindrical shell with discontinuities: Double plate bulkhead," J. Acoust. Soc. Am. 98, 339-352 (1995).

${ }^{13}$ S. Park, Sound wave scattering by cylindrical shells with internal structures (Ph. D. dissertation, Mass. Inst. Tech., Cambridge, MA, 1995).

${ }^{14}$ A. Pan, J. Fan, and B. Wang, "Acoustic scattering from a double periodically bulkheaded and ribbed finite cylindrical shell," J. Acoust. Soc. Am. 134, 3452-3463 (2013).

${ }^{15}$ D. M. Photiadis and B. H. Houston, "Anderson localization of vibration on a framed cylindrical shell," J. Acoust. Soc. Am. 106, 1377-1391 (1999).

${ }^{16}$ M. H. Marcus, B. H. Houston, and D. M. Photiadis, "Wave localization on a submerged cylindrical shell with rib aperiodicity," J. Acoust. Soc. Am. 109, 865-869 (2001).

${ }^{17}$ D. M. Photiadis, J. A. Bucaro, and B. H. Houston, "The effect of internal oscillators on the acoustic response of a submerged shell," J. Acoust. Soc. Am. 101, 895-899 (1997).

${ }^{18}$ D. M. Photiadis, B. H. Houston, E. G. Williams, and J. A. Bucaro, "Resonant response of complex shell structures," J. Acoust. Soc. Am. 108, 1027-1035 (2000).

${ }^{19}$ M. Tran-Van-Nhieu, "Scattering from a ribbed finite cylindrical shell with internal axisymmetric oscillators," J. Acoust. Soc. Am. 112, 402-410 (2002).

${ }^{20}$ C. Soize, "Probabilistic structural modeling in linear dynamic analysis of complex mechanical systems, I-Theoretical elements," La Recherche Aérospatiale (English edition) 5, 23 (1986).

${ }^{21}$ M. H. Marcus and B. H. Houston, "The effect of internal point masses on the radiation of a ribbed cylindrical shell," J. Acoust. Soc. Am. 112, 961-965 (2002).

${ }^{22}$ L. Maxit, "Scattering model of a cylindrical shell with internal axisymmetric frames by using the Circumferential Admittance Approach," Appl. Acoust. 80, 10-22 (2014). 
${ }^{23}$ V. Meyer, L. Maxit, J.-L. Guyader, and T. Leissing, "Prediction of the vibroacoustic behavior of a submerged shell with non-axisymmetric internal substructures by a condensed transfer function method," J. Sound Vib. 360, 260-276 (2016).

${ }^{24}$ M. C. Junger and D. Feit, Sound, structures, and their interaction (MIT Press, Cambridge, MA, 1972).

${ }^{25}$ F. A. Firestone, "The Mobility Method of Computing the Vibration of Linear Mechanical and Acoustical Systems: Mechanical-Electrical Analogies," J. Appl. Phy. 9, 373-387 (1938).

${ }^{26}$ V. Meyer, L. Maxit, J.-L. Guyader, T. Leissing, and C. Audoly, "A condensed transfer function method as a tool for solving vibroacoustic problems," Proc. IMechE Part C: J. Mech. Eng. Science 230, 928-938 (2016).

${ }^{27}$ S. Rubin, "Improved component-mode representation for structural dynamic analysis," AIAA J. 13, 995-1006 (1975).

${ }^{28}$ L. Maxit and J.-M. Ginoux, "Prediction of the vibro-acoustic behavior of a submerged shell non periodically stiffened by internal frames," J. Acoust. Soc. Am. 128, 137-151 (2010).

${ }^{29}$ A. W. Leissa, "Vibration of shells," Scientific and Technical Information Office, National Aeronautices and Space Administration Washington, DC, USA (1973). 